\title{
3rd ICTs and Society Meeting; Paper Session - Theorizing the Internet; Paper 6: Reconstructing the Glass Bead Game. On the Philosophy of Information
}

\author{
Rainer E. Zimmermann \& Simon M. Wiedemann
}

Munich University of Applied Sciences

rainer.zimmermann@hm.edu, simon.wiedemann@hm.edu

\begin{abstract}
A computer playable version of the Glass Bead Game in the sense of Hermann Hesse's famous novel is being presented and the various onto-epistemic as well as social implications of the game are discussed. In particular, the discussion focuses on aspects of the philosophical conceptualization of information within evolving social contexts.
\end{abstract}

Keywords: glass bead game, philosophy of information, metaphysics

We refer to the popular introduction of the game in Hesse's novel published in 1943 and quote from this. For Hesse himself, "... the Glass Bead Game is a game with all the contents and values of our culture, it plays with them, as in the times of flourishing arts a painter would have played with the colours of his palette ... The formal game strives to produce a unity and harmony which is as far as possible dense, unbroken, formally perfect. The psychological game on the other side, strives for unity and harmony, not so much in the ... perfection of ... choice, organization, mediation, and opposition of contents than by a meditation which follows each stage of the game. ... [The game] shows up as a universal language and method to express all spiritual and artistic values and concepts ..."

If we reduce this game which shall express the complete human knowledge about the world to the main fields of onto-epistemic activities, then we find that it deals explicitly with a circular arrangement of three main components, namely, with a given metaphysics, a universal perfect language, and a concrete game procedure, respectively. Essentially, these three components can be conceptualized as part of the principal vision of human beings striving for understanding and control of the world they perceive. Obviously, on the ontological level, these three components associate the conceptual categories of cognition, communication, and cooperation with each other, while on the epistemological level they achieve a similar result for the technical categories of space, network, and system.

We can give two (of several) examples for earlier approaches (and fore-runners of the Glass Bead Game) towards the practical application of mediating these two conceptual triads which at the same time implies the circularly mediated components mentioned above: 1) The Ars Magna of the Catalonian philosopher Ramón Llull (Raimundus Lullus) who arranges (around 1300) main principles (dignitates) and figures within a combinatorial generation of logic and dialectic by means of systematic permutations of possible elements. This process of generation exhibits an explicit game character: " ... per tal che hom puscha mostrar / logica e philosophar / a cels qui nin saben lati / ni arabichi." (that one can logically argue and philosophize in front of those who do not know Latin or Arabic) Obviously, this sort of game serves also the purpose of presenting a universal language accessible to everyone. [Cf. Ars brevis, Meiner, Hamburg, 1999. Quote from Compendium, 6-9.] 2) The 
Characteristica Universalis of the German philosopher Gottfried Wilhelm Leibniz who introduces (around 1700) a procedure for generating conceptual analogies derived from the Taoist I-Ging (the Book of Transformations). By doing so he achieves a combinatorial graphical system of fundamental hexagrams based on a binary system of numerical symbols on which we nowadays base our computer technology. This system is actually able to anticipate Boolean logics. (And in principle, the Heyting algebras of inutitionistic logics, more and more relevant for us within recent research, can be derived from this in turn.)

Coming back to the philosophical foundations of these approaches, we notice that what they are essentially striving for is the organization of information arising from the two conceptual triads with the long-term goal of understanding its meaning. (This is in fact what Edgar Morin calls computation in the strict sense.) By the way, note also that the first of these triads is representing the approach of the Hofkirchner group while the second is representing the approach of my own group. Hence, both of them are complementary to each other: they deal with different perspectives, but one is incomplete and cannot be understood without the other, in particular, if trying to come to grips with a stringent (future) theory of complex emergent systems.

Hence, the processing of the world itself, or alternatively, evolution as to that, can be visualized as resulting from a game in the sense of the mentioned approaches: This global game however is being played by agents which are far more fundamental than those usually dealt with (not to speak of human agents). So what we can do is to define evolution as the result produced by the cooperative games of universal autonomous agents. (The latter we can define in the sense of Stuart Kauffman.) Games are based on interaction, and interaction in turn can be defined in terms of communication. Because of the intrinsic network structure of communication, games then show up as sets of strategies, and they can be represented on directed graphs. Strategies on the other hand, are rules for a move in this game. So after all, a strategy turns out to be a path in the network of edges which spans the space of free play among the vertices representing accessible states of a given system. (This can be human persons in a particular case.) A game therefore, can be expressed as a (mathematical) category whose objects are the vertices and whose morphisms are the edges of the respective network.

As one of us (REZ) has shown at various other places, a (mathematical) category can be generalized in the following sense: A category $\mathrm{C}$ which has a terminal object and pullbacks, an initial object and pushouts, exponentials, and a subobject classifyer, is called topos. Then one can formulate the following proposition originally introduced by Steven Vickers (2004): A topos is the LindenbaumTarski algebra for a logical theory whose models are the points of a space. From this we can find as a conclusion the central idea of our approach to the Glass Bead Game: Identify the space of free play for a game by its associated topos. (Identify points of that space with propositions.)

In other words: Our game is a hexagonally organized graphical surface representing a directed network with vertices and edges, where the former are theoretical propositions, and the latter are logical implications among propositions. The idea is to find, for a given theory which shows up as a network of propositions (e.g. a model of human communication as indicated in the prototype game example displayed in the talk) new propositions which have not been in the inventory in the first place. This game combines observed data and conceptual reflexion in the two strings of a usually tree-like graph, and, by doing so, is thus able to achieve new insight and theoretical innovation by applying harmony principles to the shape of the network thus produced. Hence, the game is simulating (or emulating rather) the process of research for a chosen field which is performed in front of a background of intuition which makes it necessary to operate sometimes in a mode of creative abduction (Umberto Eco) rather than in a mode of permanent induction or deduction proper. (This replaces the meditation in Hesse's novel.) Essentially, the procedure is algorithmic, but in a generalized sense.

What we can conclude is that the game introduced here models a process of research 
which is mainly based on two activities: on the re-constructing of a network of logical implications acting upon sets of propositions, on the one hand, and on a guided intuition (creative abduction), on the other hand. This game structure is well compatible with many approaches to the human consciousness from Lévi-Strauss up to Sartre, and to the language games of Lorenzer. (Mainly, of course, with the theory of systems as proposed by Edgar Morin in his master piece on "The Method".) The underlying concept of harmony (the game's objective in Hesse) is primarily determined by its correspondence with the two conceptual triads arising in the theory of complex emergent systems (what in Hofkirchner's terminology is the theory of evolutionary systems).

Obviously, the game puts forward a strictly interdisciplinary perspective (not restricted to the sciences, but also encompassing the arts). Perhaps this is what connects us with our colleague Ropolyi. And in particular, the field of physics (constitutive for our modern understanding of sciences) - as well as all the rest - shows up here as the cognitively accessible manifestation of the permanent (and practically universal) processing of information in the sense of Morin's computation. This might place us more in the vicinity of Blanchette rather than in opposing distance. As to the ethical viewpoint which is apparently immanent in all contributions to this section here, we may find explicit criteria by visualizing the achievable harmony in terms of an observable identity shape which is nothing but the outcome of processing difference within the triadic framework discussed above. Hence, our game illustrates an approach to the world which is based on the dialectical identity of identity and difference in the tradition of Schelling. On a fundamental level then, reconstructing the Glass Bead Game means reconstructing the human mode of being. (More on this can be found in our forthcoming book which will be published in 2010 in German: "Topos der Materie. Neue Anleitung zum Glasperlenspiel." Shaker, Aachen.) 